\title{
A importância do acompanhamento clínico de lesões brancas potencialmente malignas em mucosa labial: relato de caso
}

The importance of clinical follow-up of potentially malignant white lesions on the labial mucosa: case report La importancia del seguimiento clínico de lesiones blancas potencialmente malignas en mucosa labial: relato de caso

\section{Vanessa EINSFELD ${ }^{1}$ \\ Ana Claudia RAMOS ${ }^{1}$ \\ Beatriz BARBOSA ${ }^{1}$}

Alberto ZEN ${ }^{1}$

Grasieli de Oliveira RAMOS ${ }^{2,3}$

Acir José DIRSCHNABEL ${ }^{2}$

${ }^{1}$ Cirugião-dentista, Faculdade de Odontologia, UNOESC, 89600-000, Joaçaba-SC, Brasil

${ }^{2}$ Professor, Faculdade de Odontologia, UNOESC, 89600-000, Joaçaba-SC, Brasil

${ }^{3}$ Professor, Mestrado em Biociências e Saúde, UNOESC, 89600-000, Joaçaba-SC, Brasil

\section{Resumo}

Introdução: Quando estamos frente às lesões potencialmente malignas, torna-se imprescindível o acompanhamento rigoroso dessas lesões, pois são lesões frequentes e com risco de transformação maligna consideravelmente relevante, a leucoplasia e a queilite actínica, são as lesões mais comuns encontradas na cavidade bucal. Objetivo: relatar um caso de lesão potencialmente maligna em mucosa labial. Relato de caso: Paciente sexo masculino compareceu à clínica de Diagnóstico VI da Universidade do Oeste de Santa Catarina, com a presença de lesão branca em lábio inferior, o mesmo já se encontrava em acompanhamento há seis anos, com diagnóstico prévio de queilite actínica sem atipia celular (duas biópsias prévias). Aposentado, trabalhava anteriormente como agricultor, ex-fumante e ex-etilista. Clinicamente observava-se lesão esbranquiçada, com aspecto verruciforme, localizada em lábio inferior, medindo $2 \mathrm{~cm}$ no maior diâmetro. Além disso, o lábio encontrava-se endurecido, com aspecto roliço. Duas hipóteses de diagnóstico foram levantadas: queilite actínica associada a leucoplasia e carcinoma verrucoso. O paciente foi submetido à nova biópsia incisional. No laudo constatou-se tratar de queilite actínica com displasia epitelial leve e o tratamento proposto foi o uso de protetor labial FPS30, além de acompanhamento clínico semestral. Conclusão: O acompanhamento desse caso clínico foi possível observar alteração no perfil citológico, onde nas primeiras biópsias não era possível observar atipia celular e na biópsia mais recente foi observado uma displasia epitelial leve, portanto o acompanhamento das lesões potencialmente malignas é fundamental para prevenir sua transformação maligna, e uma nova biópsia deve ser realizada sempre que for observada alteração no aspecto clínico da lesão.

Descritores: Leucoplasia; Queilite; Biópsia; Lábio.

\section{Abstract}

Introduction: When we are facing potentially malignant lesions, it is essential to do a strictly follow up of these lesions, since they are frequent and with a significant malignant transformation risk. Leukoplakia and actinic cheilitis are the most common potentially malignant lesions found in the oral cavity. Objective: report a case of potentially malignant lesion on the labial mucosa. Case report: Male patient was referred to Clinic oral of Diagnosis at University of the West of Santa Catarina, with a white lesion in lower lip. The patient had been following the lesion for six years. The previous diagnosis was actinic cheilitis without dysplasia (two previous incisional biopsies). Retired, previously worked as a farmer, ex-smoker and ex-alcoholist. The clinical evaluation revealed a whitish, verrucciform-like plake, measuring 2 $\mathrm{cm}$ in diameter, localized in lower lip. The lip was a hardness and plump. The diagnosis hypothesis was actinic cheilitis associated with leukoplakia and verrucous carcinoma. New incisional biopsy was done and the histopathological analysis reveals an actinic cheilitis with mild epithelial dysplasia. To treatment, we proposed the use of FPS30 lip balm, and to biannual clinical follow-up. Conclusion: During the clinical follow up, we can note the change in cytological profile, in the firsts biopsies we didn't have cytological atipia, but in the recent biopsy we can observe mild epithelial dysplasia. Because of that, the clinical follow up of these lesions is fundamental to prevent the malignant transformation, and a new biopsy must be made always when we observe changes in clinical aspect of the lesion.

Descriptors: Leukoplakia; Cheilitis; Biopsy; Lip.

\section{Resumen}

Introducción: Cuando estamos frente a las lesiones potencialmente malignas, se hace imprescindible el acompañamiento riguroso de esas lesiones, pues son frecuentes y con riesgo de transformación maligna considerablemente relevante, la leucoplasia y la queilitis actínica, son las lesiones potencialmente malignas más comunes encontradas en la cavidad bucal. Objetivo: relatar un caso de lesión potencialmente maligna en mucosa labial. Relato de caso: paciente sexo masculino, compareció a la clínica de diagnóstico oral de la Universidad del Oeste de Santa Catarina, con una lesión blanca en labio inferior. El paciente ya se encontraba en acompañamiento de la lesión hace 6 años, con diagnóstico previo de queilitis actínica sin atipia celular (dos biopsias previas). Retirado, pero trabajaba como agricultor, ex fumador y exalcohólica. Durante la evaluación clínica fue observada una lesión blancuzca, con aspecto verrugoso, de $2 \mathrm{~cm}$ en el mayor diámetro, localizada en labio inferior. Además, el labio se encontraba endurecido, con aspecto rodillo. Dos hipótesis de diagnóstico fueron planteadas: queilitis actínica asociada a leucoplasia y carcinoma verrugoso. El paciente fue sometido a nueva biopsia incisional. En el laudo se constató tratar de queilitis actínica con displasia epitelial leve y el tratamiento propuesto fue el uso de protector labial FPS30, además de acompañamiento clínico semestral. Conclusión: El acompañamiento clínico de este caso fue posible observar la alteración del perfil citológico, donde el las primeras biopsias no era posible observar atipia celular y en la biopsia más reciente fue observado displasia epitelial leve, por lo tanto el acompañamiento de las lesiones potencialmente malignas es fundamental para prevenir su transformación maligna, y una nueva biopsia debe ser realizada siempre que sean observadas mudanzas en el aspecto clínico de la lesión.

Descriptores: Leucoplasia; Queilitis; Biopsia; Labio.

INTRODUÇÃO

Ao examinar um indivíduo, deve-se ficar atento a lesões precursoras ao carcinoma oral, observando alterações da mucosa como as lesões brancas, cuja coloração acinzentada ou esbranquiçada contrasta com a coloração róseo-avermelhada da mucosa normal ${ }^{1}$.

É definido como lesão potencialmente maligna qualquer tecido benigno, morfologicamente alterado, que apresente um maior risco do que o normal de transformação maligna. Entre as lesões com potencialmente malignas caracterizadas por placas brancas destacasse a leucoplasia, a queilite actínica e o líquen plano oral ${ }^{2-4}$.

Em 1978, a Organização Mundial de Saúde (OMS) propôs pela primeira vez a definição de leucoplasia como "uma placa ou mancha branca que 
não pode ser caracterizada clínica e patologicamente como qualquer outra doença" o termo é estritamente clinico e não implica uma alteração tecidual histopatológica especifica ${ }^{3}$. O termo foi adaptado diversas vezes, sendo sua última versão, como "placas brancas de malignização possível após exclusão daquelas que não transportam tal risco"4.

Seu aparecimento ocorre principalmente em homens acima dos 40 anos, encontradas em aproximadamente $70 \%$ dos casos no vermelhão do lábio, seguidos da mucosa oral e gengivas. Das lesões em língua, assoalho de boca e vermelhão dos lábios, $90 \%$ exibem displasias ou carcinoma ${ }^{3}$. A causa das leucoplasias é desconhecida, contudo existem algumas teorias sobre o fator etiológico causador. Uma das teorias mais aceitas relata que o uso do tabaco estaria intimamente ligado ao seu aparecimento, sendo que mais de $80 \%$ dos pacientes com leucoplasia intraorais são tabagistas. Outra hipótese associa a radiação ultravioleta como o fator etiológico da leucoplasia de vermelhão do lábio inferior, estando diretamente relacionada à queilite actínica $^{3,5,6}$.

A queilite actínica (QA) é uma alteração com potencial de malignização comum do vermelhão do lábio inferior (95\% dos casos), que resulta de uma exposição progressiva e/ou excessiva aos raios ultravioletas (UV). Ocorre geralmente em homens em uma proporção de 10:1, acima dos 45 anos, leucoderma, com ocupação profissional ao ar livre. Sua malignização pode resultar no carcinoma espinocelular, sendo que este carcinoma corresponde a aproximadamente $94 \%$ de todas as lesões malignas da mucosa bucal ${ }^{3,4,7}$.

O diagnóstico da queilite actínica é baseado em uma avaliação clínica e histopatológica. Há divergência com relação à necessidade de biópsia. Alguns autores relatam que a necessidade de biópsia vai de acordo com a evolução da lesão, porém alguns autores afirmam que a biópsia é indicada em todos os casos, pois há grande risco de malignização ${ }^{8}$. Já o diagnóstico das leucoplasias é dado por exclusão, ou seja, a partir de uma avaliação clínica, realiza-se uma biópsia da lesão, após analisar o laudo histopatológico, onde constará a exclusão de outras placas brancas semelhantes, dá-se o diagnóstico de leucoplasia, já que esta não pode ser caracterizada clinicamente ou patológicamento como qualquer outra lesão ${ }^{3,9}$.

No presente estudo será relatado o caso de um paciente atendido na clínica de Diagnóstico VI da Universidade do Oeste de Santa Catarina (UNOESC), com acompanhamento durante seis anos de placa branca em lábio inferior.

\section{CASO CLÍNICO}

Paciente, sexo masculino, leucoderma, 60 anos de idade, aposentado e motorista, trabalhava anteriormente como agricultor, ex-fumante (abstêmio há 12 anos) e ex-etilista (abstêmio a 7 anos), compareceu a clínica de Diagnóstico VI da Universidade do Oeste de Santa Catarina (UNOESC) em 2016, para acompanhamento de lesão branca anteriormente diagnosticada. Foi verificado que o paciente já havia realizado duas biópsias incisionais com diagnóstico de queilite actínica sem atipia celular $(2010$ e 2013). Na época foi proposto o tratamento com o uso de protetor solar FBS $30 \mathrm{em}$ bastão. No exame clínico atual observou-se lesão esbranquiçada, com aspecto verruciforme, medindo 2 $\mathrm{cm}$ no maior diâmetro, localizada em lábio inferior (Figura 1). Além disso, o lábio encontrava-se endurecido, com aspecto roliço. Duas hipóteses de diagnóstico foram levantadas: queilite actínica associada a leucoplasia e carcinoma verrucoso.

Diante das características clínicas da lesão, histórico do paciente e tamanho da lesão optou-se pela realização de biópsia incisional. A biópsia foi realizada através de uma incisão superficial em forma de cunha, abrangendo tecido epitelial e conjuntivo. Para conseguir uma melhor aproximação das bordas durante a sutura e minimizar os danos estéticos foi realizado uma manobra cirúrgica chamada divulsão do tecido (Figura 1).

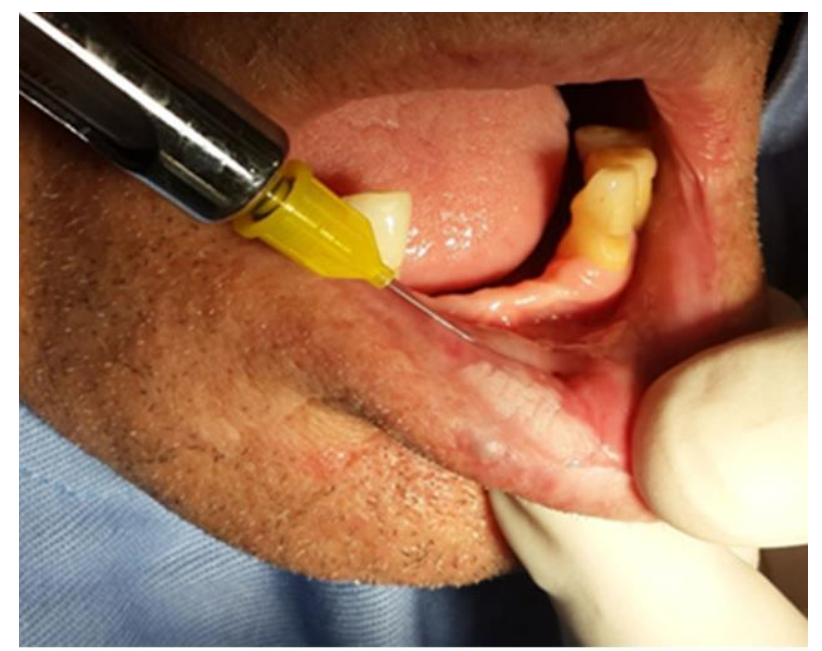

Figura 1: Lesão inicial evidenciando placa branca, com aspecto verrucoso, durante biópsia incisional.

O exame histopatológico (Figura 2) revelou tecido epitelial de revestimento pavimentoso estratificado exibindo abundante hiperceratose (imagem maior), com alterações de maturação e proliferação epitelial restritas ao terço inferior do epitélio (imagem menor). Além disso, o tecido conjuntivo denso apresentava intensa elastose solar (seta), concluindo-se tratar de queilite actínica com displasia epitelial leve. No pós-operatório de 30 dias observou-se boa cicatrização da área operada (Figura 3). O tratamento proposto foi o uso de filtro solar FPS 30 em bastão, além do uso de chapéu de aba larga. Também foi proposto, acompanhamento semestral para controle da evolução da lesão, e para estabelecer um contato mais próximo com o paciente. 


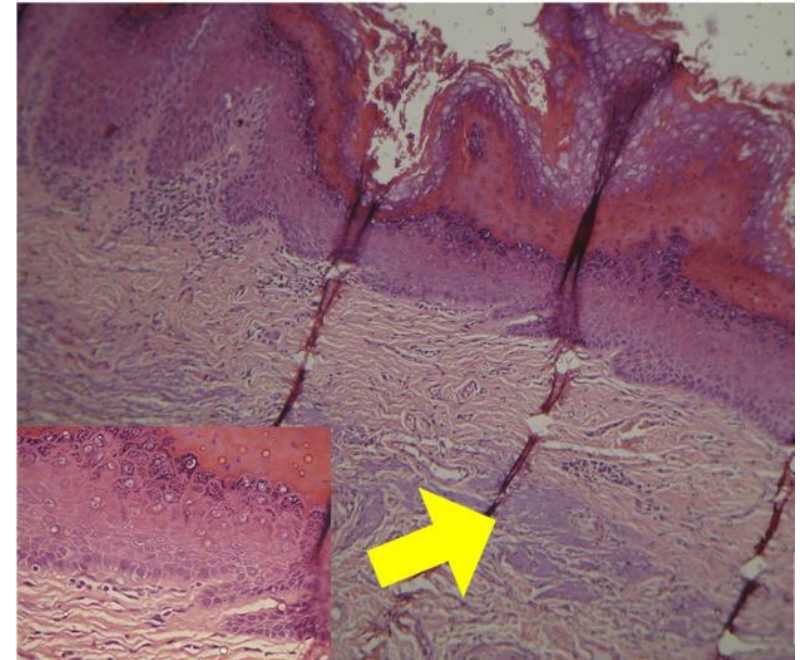

Figura 2: Imagem histopatológica mostrando abundante hiperceratose (imagem maior), displasia epitelial (imagem menor) e elastose solar (seta).

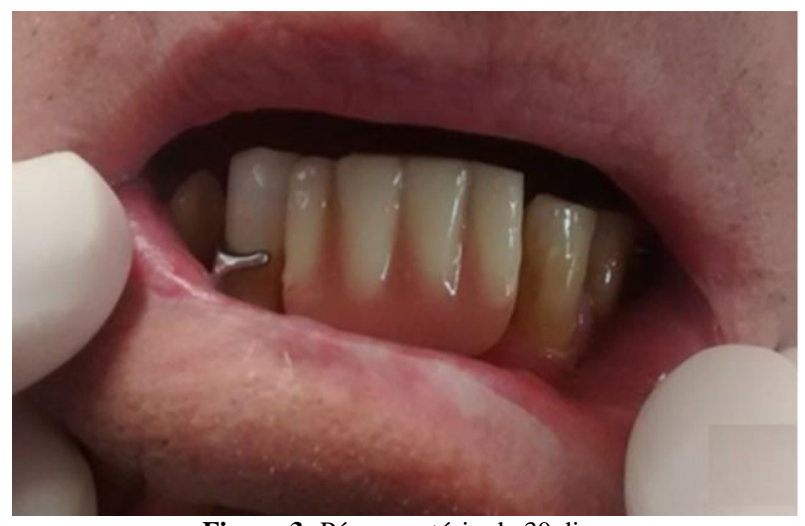

Figura 3: Pós-operatório de 30 dias

O paciente encontra-se em acompanhamento e apresenta-se livre de recorrência, permanecendo apenas a lesão remanescente da lesão inicial (Figura 4).

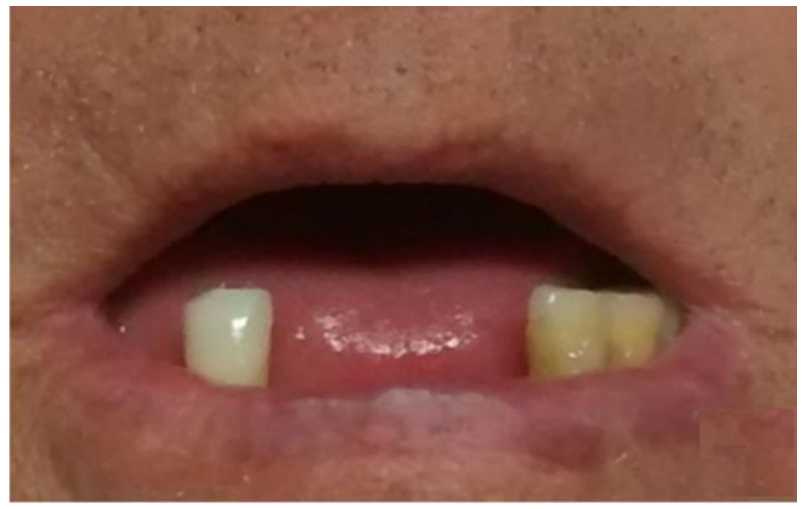

Figura 4: Acompanhamento clínico de 120 dias.

\section{DISCUSSÃO}

O termo lesões potencialmente malignas determina áreas de tecido com alterações clínicas e morfológicas com potencial para malignizar. Além disso, algumas delas (lesões brancas e vermelhas) ocorriam frequentemente nas margens dos carcinomas espinocelular orais. Outras partilhavam alguns dos aspectos das lesões epiteliais malignas, mas sem invasão do tecido conjuntivo. Estudos mostraram que entre 16 e $62 \%$ dos carcinomas orais, são diagnosticados clinicamente como lesões leucoplásicas ${ }^{10-14}$. Além disso, alterações cromossómicas, genômicas e moleculares, características dos carcinomas invasivos, podem também ser detectadas nas lesões potencialmente malignas, assim mostrando a importância do diagnóstico precoce dessas lesões ${ }^{4}$.

À medida que a lesão de QA progride, áreas ásperas e recobertas de escamas desenvolvem-se nas porções mais secas do vermelhão. Essas áreas se tornam espessas e podem se assemelhar a lesões leucoplásicas, especialmente quando se estendem para próximo da linha úmida do lábio, o que justifica um possível erro de diagnóstico e a necessidade de uma biópsia ser realizada ${ }^{3}$.

As alterações associadas à QA podem ser irreversíveis, porém os pacientes devem ser encorajados a utilizar bloqueadores solares para lábios para evitar a progressão da lesão. As áreas de espessamento, endurecimento, leucoplasia ou ulceração devem ser submetidas à biópsia para realizar o diagnóstico definitivo dessas lesões e excluir a possibilidade de já ser um carcinoma espinocelular. O tratamento da QA depende do grau de alterações, nos casos mais leves pode-se realizar o acompanhamento clínico além do uso de bloqueadores solares, já nos casos clinicamente graves sem transformação maligna clara, a vermelhectomia deve ser realizada. Nessa manobra a mucosa do vermelhão é removida e uma porção da mucosa labial intraoral é puxada em direção à ferida ou permite-se que a ferida cicatrize por segunda intenção. A vantagem dessa técnica é a possibilidade de analisar microscopicamente todo o tecido removido, nessa análise pode-se observar ausência de malignidade, displasia epitelial nos diferentes graus ou carcinoma espinocelular invasivo. Além disso outros tratamentos alternativos também são sugeridos, como a ablação a laser de $\mathrm{CO} 2$ ou de érbio: YAG (Er: YAG \{érbio: ítrio, alumínio\}), eletrodissecção, aplicação tópica de imiquimod (quimioterápico tópico usado para tratar tumores na pele, como carcinoma basocelular, verruga anogenital e queratose), terapia fotodinâmica e quimioexfoliação (ou peeling químico) com ácido tricloroacético. Independente do tratamento realizado um acompanhamento de longa duração é recomendado ${ }^{3}$.

A QA é uma lesão que merece atenção especial, pois, como se sabe, pertence ao grupo de lesões potencialmente malignas, considerada por alguns autores como precursora do carcinoma espinocelular do lábio inferior ${ }^{1,4,7,15}$. Uma vez que esse carcinoma se desenvolve a invasão linfática é muito mais rápida do que na pele. $\mathrm{O}$ carcinoma espinocelular do lábio metatastiza mais frequentemente do que os análogos da pele e da cavidade oral, com uma porcentagem de 3 a $20 \%$ para carcinoma de lábio e de 0,5 a $3 \%$ para o de pele. Porém, o prognóstico deste carcinoma é melhor 
quando em comparação com os carcinomas intraorais $^{16-18}$.

As etapas de transformação maligna da leucolasia envolvem várias alterações genéticas, resultado da inativação de genes supressores de tumor e ativação de proto-oncogenes, que são causadas por pontos de mutação, amplificação gênica, promoção de metilações e deleções. Pesquisas revelam que em tecidos acometidos por leucoplasia, principalmente os que apresentam displasia epitelial, exibem alterações moleculares no gene supressor WWOX (domínio oxidante contendo oxidoredutase), uma alteração genética precoce que pode contribuir para carcinogênese oral, embora outras pesquisas não concordarem com essa hipótese $^{19}$. As estimativas de malignização destas lesões variam muito dependendo da localização na cavidade oral, da população estudada e de suas etnias. Essas variações podem ser atribuídas a diferentes tempos de acompanhamento, grupos de estudo e hábitos do paciente ${ }^{20}$.

As lesões de leucoplasia também exigem cuidado especial. Após a biópsia com a confirmação de ausência de malignidade pode-se realizar a remoção completa ou optar-se por realizar o acompanhamento clínico. Lesões com alterações displásicas podem ser removidas na sua totalidade, especialmente as que apresentarem graus mais altos de displasia. Essa remoção pode ser realizada com igual eficiência pela excisão cirúrgica, eletrocauterização, criocirurgia ou ablação por laser. Um acompanhamento de longa duração após remoção é de extrema importância, pois as recidivas são frequentes e também porque outras leucoplasias podem se desenvolver, principalmente se os hábitos não forem alterados. As recorrências ocorrem especialmente para os tipos verruciforme ou granular, onde $83 \%$ deles recidivam e requerem remoção adicional. As leucoplasias que não exibem displasia geralmente não são excisadas, porém uma avaliação clínica a cada 6 meses é recomendada devido a possibilidade de alteração do diagnóstico histopatológico para uma displasia epitelial. Outras biópsias são recomendadas sempre que o hábito de fumar continue ou ocorreram alterações clínicas significantes $^{3}$.

A coloração com azul de toluidina pode ser utilizada rotineiramente como método para escolha do melhor sitio de biópsia e para acompanhamento de lesões potencialmente malignas. Células atípicas apresentam maior conteúdo de material genético e como o azul de toluidina tem maior afinidade por material nuclear com alto conteúdo de DNA ou RNA, áreas que apresentam estas alterações têm a capacidade de reter maior quantidade de corante. Autores sugerem que a coloração escura do teste com azul de toluidina demonstrou um resultado positivo verdadeiro e mostrou que a intensidade da coloração está relacionada à severidade da lesão ${ }^{21-23}$.

As lesões potencialmente malignas podem preceder o carcinoma espinocelular bucal. Ele é a neoplasia maligna mais prevalente da cavidade oral, representando $95 \%$, desses apenas $15 \%$ de todas as ocorrências são diagnosticadas em estágios iniciais e $50 \%$ dos pacientes procuram tratamentos em estágios avançados com metástases já instaladas. Por isso as lesões potencialmente malignas são manifestações de grande importância devido a possibilidade de diagnóstico precoce de câncer e minimizando todas as suas implicações ${ }^{24}$.

\section{CONCLUSÃO}

É de responsabilidade do cirurgião dentista fazer uma minuciosa anamnese e inspeção clínica, para que lesões potencialmente malignas sejam diagnosticadas em uma fase inicial. É imprescindível a necessidade de biópsia nestes casos, para que se obtenha um diagnóstico correto, além de um planejamento de tratamento eficaz. As lesões com potencial de malignização devem receber uma atenção especial, fazendo um acompanhamento semestral da lesão.

\section{REFERÊNCIAS}

1. Pindborg JJ, Reichart PA, Smith CJ, Van der Waal I. World Health Organization International histological classification of tumours. Histological typing of câncer and precancer of the oral mucosa. Berlin: Springer; 1997.

2. Marley JJ, Linden GJ, Cowan CG, Lamey PJ, Warnakulasuriya KAAS, Scully C. Management of potentially malignant oral mucosa lesions by consultant UK oral and maxillofacial surgeons. Br J Oral Maxillofac Surg. 1996;34(1):28-36.

3. Neville BW, Dam DD, Allen CM, Chi AC . Patologia oral e maxilofacial. 3. ed. Rio de Janeiro; 2016.

4. Warnakulasuriya S, Johnson NW, van der Waal I. Nomenclature and classification of potentially malignant disorders of the oral mucosa. J Oral Pathol Med. 2007;36(10):575-80.

5. Gupta PC, Murti PR, Bhonsle RB, Mehta FS, Pindborg JJ. Effect of cessation of tobacco use on the incidence of oral mucosal lesions in a 10-yr follow-up study of 12.212 users. Oral Dis. 1995;1(1):54-8.

6. Maito FDM. Avaliação da expressão do PCNA no epitélio lingual de camundongos submetidos à ingestão e aplicação tópica de álcool a $40 \mathrm{GL}$ [dissertação]. Porto Alegre: Faculdade de Odontologia - UFRGS;2001.

7. Markopoulos A, Albanidou-Farmaki E, Kayavis I. Actinic cheilitis: clinical and pathologic characteristics in 65 cases. Oral Dis. 2004;10(4):212-16.

8. Cintra JS, Torres SCM, Silva MBF, Manhães Júnior LRC, Silva Filho JP, Junqueira JLC. 
Queilite Actínica: estudo epidemiológico entre trabalhadores rurais no município de ParacaiaSP. Ver Assoc Paul Cir Dent. 2013;67(2):118-21.

9. Greespan D, Jordan RCK. The white lesions that kills - aneuploide dysplastic oral leukoplakia. N Engl J Med. 2004;350(14):1382-84.

10. Bánóczy J. Follow-up studies in oral leukoplakia. J Maxillofac Surg. 1977;5(1):69-75.

11. Huber MA. White oral lesions, actinic cheilitis, and leukoplakia: confusions in terminology and definition: facts and controversies. Clin Dermatol. 2010;28(3):262-68.

12. Reibel J. Prognosis of oral pre-malignant lesions: significance of clinical, histopathological and molecular biological characateristics. Crit Rev Oral Biol Med. 2003;14(1):47-62.

13. Scheifele C, Reichart PA, Oral leukoplakia in manifest squamous epithelial carcinoma. A clinical prospective study of 101 patients, Mund Kiefer Gesichtschir. 1998;2(6):326-30.

14. Schepman K, der Meij E, Smeele L, der Waal I. Concomitant leukoplakia in patients with oral squamous cell carcinoma. Oral Dis. 1999;5(3):206-9.

15. Kaugars GE, Pillion T, Svirsky JA, Page DG, Burns JC, Abbey LM. Actinic cheilitis: a review of 152 cases. Oral Surg Oral Med Oral Pathol Oral Radiol Endod. 1999;88(2):181-86.

16. Abreu MAMA, Silva OMP, Pimentel DRN, Hirata CHW, Weckx LLM, Alchorne MMA et al. Actinic cheilitis adjacent to squamous carcinoma of the lips as an indicator of prognosis. Braz $\mathbf{J}$ Otorhinolaryngol. 2006;72(6):767-71.

17. Patrício JFC. Evolução das lesões pré-malignas orais: orientações para os médicos dentistas [dissertação]. Porto: Universidade do Porto; 2011.

18. Shah AY, Doherty SD, Rosen T. Actinic cheilitis: a treatment review. Int J Dermatol. 2010; 49(11):1225-34.

19. Pimenta FJ, Cordeiro GT, Pimenta LGGS, Viana MB, Lopes J, Gomez MV et al. Molecular alterations in the tumor suppressor gene WWOX in oral Leukoplakias. Oral Oncol. 2008;44(8):753-58

20. Paulo LFB, Rosa RR, Rocha MA, Durighetto Junior AF. Incidência e prevalência das lesões brancas associadas ao tabagismo atendidos no ambulatório da Unidade de Diagnóstico Estomatológico da Universidade Federal de Uberlândia no período de 1997 a 2008. Horizonte Cientifico. 2011;2:1-20.

21. Gandolfo S, Pentenero M, Broccoletti R, Pagano M, Carrozzo M, Scully C. Toluidine blue uptake in potentially malignant oral lesions in vivo: clinical and histological assessment. Oral Oncol. 2006;42(1):89-95.

22. Mashberg A, Samit A. Early diagnosis of asympomatic oral and oropharyngeal squamous cancers. CA Cancer J Clin. 1995;45(6):328-51.
23. Mendes SF, Ramos GO, Rivero ERC, Modolo F, Grando LJ, Meurer MI. Techniques for precancerous lesion diagnosis. J Oncol. 2011. ID 326094.

24. Dib LL, Kowalski LP, Curi MM. Lesões cancerizáveis de boca. In: Kowaski LP, Anelli A, Salvajoli JV, Lopes LF. Manual de condutas diagnosticas e terapêuticas em oncologia. 2.ed. São Paulo: Âmbito Editores; 2002.

\section{CONFLITO DE INTERESSES}

Os autores declaram não haver conflitos de interesse.

\section{AUTOR PARA CORRESPONDENCIA}

Acir José Dirschnabel

acirdirschnabel@unoesc.edu.br

Submetido em 09/03/2019

Aceito em 11/06/2019 